\title{
ISOLATED, MASSIVE SUPERGIANTS NEAR THE GALACTIC CENTER
}

\author{
M. P. Muno, ${ }^{1,2}$ G. C. Bower, ${ }^{3}$ A. J. Burgasser, ${ }^{4,5,6}$ F. K. Baganoff, ${ }^{7}$ M. R. Morris, ${ }^{1}$ and W. N. Brandt ${ }^{8}$ \\ Received 2005 July 1; accepted 2005 October 6
}

\begin{abstract}
We have carried out a pilot project to assess the feasibility of using radio, infrared, and X-ray emission to identify young, massive stars located between 1 and $25 \mathrm{pc}$ from the Galactic center. We first compared catalogs compiled from the VLA, Chandra, and 2MASS. We identified two massive, young stars: the previously identified star that is associated with the radio H II region H2 and a newly identified star that we refer to as CXOGC J174516.1-290315. The infrared spectra of both stars exhibit very strong $\mathrm{Br} \gamma$ and $\mathrm{He}$ I lines and resemble those of massive supergiants that have evolved off of the main sequence but not yet reached the Wolf-Rayet phase. We estimate that each star has a bolometric luminosity $\gtrsim 10^{6} L_{\odot}$. These two stars are also associated with bright mid-infrared sources from the MSX survey, although the origin of this emission is uncertain. Likewise, the detection of these two sources in X-rays is surprising because stars at similar evolutionary states are not uniformly bright X-ray sources. Therefore, we suggest that both stars are in binary systems that contain either OB stars whose winds collide with those of the luminous supergiants or compact objects that are accreting from the winds of the supergiants. We also identify X-ray emission from a nitrogentype Wolf-Rayet star and place upper limits on the X-ray luminosities of three more evolved, massive stars that previously have been identified between 1 and $25 \mathrm{pc}$ from Sgr A*. Finally, we briefly discuss the implications that future searches for young stars will have for our understanding of the recent history of star formation near the Galactic center.
\end{abstract}

Subject headings: Galaxy: center — infrared: stars — radio continuum: stars — stars: emission-line, $\mathrm{Be}$ X-rays: stars

\section{INTRODUCTION}

In contrast to the Galactic bulge, the inner $300 \mathrm{pc}$ of the Galaxy is experiencing ongoing star formation. This is dramatically displayed by the $\gtrsim 60$ ultracompact $\mathrm{H}$ II regions in the giant molecular cloud Sgr B2 (de Pree et al. 1998) and by three young, dense clusters of massive stars (the Arches, the Quintuplet, and the central parsec; e.g., Krabbe et al. 1995; Figer et al. 1999). The average star formation rate is estimated to be $\sim 0.02 M_{\odot} \mathrm{yr}^{-1}$, or $\sim 1 \%$ of the total Galactic value (Figer et al. 2004). About half of the recent star formation can be accounted for by the three massive star clusters mentioned above, but this implies that the sites of formation for the other half of the young stars in the central $300 \mathrm{pc}$ have not yet been identified. One possibility is that star clusters less massive than the Arches and Quintuplet $\left(\sim 10^{4} M_{\odot}\right)$ have already begun to dissipate and are difficult to identify against the dense background of older stars near the Galactic center (Portegies-Zwart et al. 2002). Alternatively, a half-dozen relatively isolated H II regions near the Galactic center have been associated with individual young, massive stars (Cotera et al. 1999), which could suggest that stars can also be formed in small associations. Our

\footnotetext{
${ }^{1}$ Department of Physics and Astronomy, University of California, Los Angeles, CA 90095; mmuno@astro.ucla.edu.

2 Hubble Fellow.

3 Radio Astronomy Lab, University of California, Berkeley, CA 94720.

4 Department of Astrophysics, Division of Physical Sciences, American Museum of Natural History, Central Park West at 79th Street, New York, NY 10024.

${ }_{5}^{5}$ Spitzer Fellow.

6 Visiting Astronomer at the Infrared Telescope Facility, which is operated by the University of Hawaii under cooperative agreement NCC 5-538 with the National Aeronautics and Space Administration, Office of Space Science, Planetary Astronomy Program.

${ }^{7}$ Kavli Institute for Astrophysics and Space Research, Massachusetts Institute of Technology, Cambridge, MA 02139.

8 Department of Astronomy and Astrophysics, Pennsylvania State University, University Park, PA 16802.
}

understanding of how stars form near the Galactic center would be greatly helped by identifying more of the young stars expected to exist there.

We have carried out a radio survey at $8.4 \mathrm{GHz}$ with the Very Large Array (VLA) ${ }^{9}$ of the region between $3^{\prime}$ and $10^{\prime}$ from the supermassive black hole Sgr $\mathrm{A}^{*}$ in order to identify counterparts to the $\approx 2000 \mathrm{X}$-ray sources identified using the Chandra $X$-Ray Observatory (Muno et al. 2003). The general properties of the radio sources detected in this survey will be described elsewhere. Here we report the properties of two radio and X-ray sources that also had counterparts among the $\approx 18,000$ stars in the same region from the Two Micron All Sky Survey (2MASS; $\S 2.1$ ). We identify one, CXOGC J174516.1-290315, as a new emission-line star located $10 \mathrm{pc}$ in projection from the Galactic center. ${ }^{10}$ The second is a previously identified luminous young star associated with the radio $\mathrm{H}$ II region $\mathrm{H} 2$ (Yusef-Zadeh \& Morris 1987; Zhao et al. 1993; Figer 1995; Cotera et al. 1999).

We also present an assessment of whether X-ray and radio surveys could be generally effective for identifying luminous young stars $(\S 2.2)$. We find X-ray emission from two of five massive, emission-line stars that were previously identified through infrared spectroscopy of stars located near $\mathrm{H}$ II regions by Cotera et al. (1999) (H2 and a WN 6 star associated with Sgr A-A) and provide updated positions for all five stars based on the 2MASS catalog. We discuss the origin of the X-ray emission from the young stars in our sample and the implications

\footnotetext{
9 The VLA is operated by the National Radio Astronomy Observatory, which is a facility of the National Science Foundation, operated under cooperative agreement by Associated Universities, Inc.

${ }^{10}$ We note that CXOGC J174516.1-290315 was previously identified as a candidate young stellar object (YSO) using data from the ISOGAL survey (Felli et al. 2002). In their catalog, it is referred to as ISOGAL-P J174516.2-290315. Our identification of the source suggests that the mid-infrared colors and fluxes that they used to select YSOs also selects hot, post-main-sequence stars.
} 
TABLE 1

Basic Properties of Young Stars Near the Galactic Center

\begin{tabular}{|c|c|c|c|c|c|c|c|c|}
\hline NAME & $\begin{array}{l}\text { R. A. (J2000.0) } \\
\text { (deg) }\end{array}$ & $\begin{array}{l}\text { DeCL. (J2000.0) } \\
\quad(\mathrm{deg})\end{array}$ & $\begin{array}{c}\text { OFFSET } \\
\text { (arcmin) }\end{array}$ & $J$ & $H$ & $K_{s}$ & \multicolumn{2}{|c|}{ X-Ray Counts } \\
\hline X174516.1........... & 266.31744 & -29.05434 & 5.9 & $11.49 \pm 0.02$ & $9.12 \pm 0.03$ & $7.89 \pm 0.02$ & $706 \pm 31$ & $170 \pm 4$ \\
\hline $\mathrm{H} 2 \ldots \ldots \ldots \ldots \ldots$ & 266.36924 & -28.93472 & 5.0 & 14.3 & $11.26 \pm 0.03$ & $9.22 \pm 0.03$ & $201 \pm 18$ & $86 \pm 2$ \\
\hline 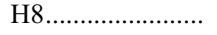 & 266.40761 & -28.95448 & 3.2 & $15.6 \pm 0.1$ & $12.30 \pm 0.08$ & $10.30 \pm 0.05$ & $<17$ & $52 \pm 1$ \\
\hline 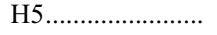 & 266.41381 & -28.88923 & 7.1 & $14.73 \pm 0.04$ & $11.68 \pm 0.04$ & $10.19 \pm 0.05$ & $<27$ & $257 \pm 7$ \\
\hline Sgr A-A................... & 266.46089 & -28.98879 & 2.6 & $15.59 \pm 0.07$ & $12.45 \pm 0.02$ & $10.90 \pm 0.03$ & $52 \pm 12$ & $80 \pm 2$ \\
\hline
\end{tabular}

NotEs.-Positions are accurate to 0.3 . The offset is relative to Sgr A*. Uncertainties on the infrared magnitudes are provided where they are available from the 2MASS catalog.

that the detection of the new young, massive star has for understanding the star formation history near the Galactic center $(\S 3)$.

\section{OBSERVATIONS}

\subsection{Radio- and X-Ray-Selected Young Stars}

We identified two young stars by comparing a catalog of discrete sources compiled from VLA observations at $8.4 \mathrm{GHz}$ (3.6 cm; G. C. Bower et al. 2006, in preparation) to the Chandra and 2MASS catalogs. The VLA observations were taken in the B configuration during 2002 August. They were sensitive to sources as faint as $250 \mu \mathrm{Jy}$ that were located between $5^{\prime}$ and $10^{\prime}$ of Sgr $\mathrm{A}^{*}$, although the sensitivity declined to $\approx 3 \mathrm{mJy}$ within a few arcminutes of Sgr $\mathrm{A}^{*}$ because of the bright diffuse radio emission there. The VLA observations identified 30 unresolved sources and 55 extended sources. The positions of the unresolved sources were accurate to 0 "' 1 .

We searched for X-ray counterparts using the catalog in Muno et al. (2003). The positions of the X-ray sources in the Chandra catalog are accurate to 0.3 at the aim point of the observation (i.e., Sgr A*), but within $5^{\prime}-10^{\prime}$ from Sgr A* the average uncertainty was $\sim 1^{\prime \prime}$. Since most of the radio sources were found at larger offsets, we searched for X-ray and radio sources that were located within $1^{\prime \prime}$ in projection. We found two matches between the unresolved radio sources and the X-ray catalog within a radius of $1^{\prime \prime}$. By adding random shifts to the relative alignments of the two catalogs, we determined that one of these matches was likely to be spurious. Within the extents of the diffuse VLA sources, we find five X-ray counterparts, although three or four should be random associations.

We then compared the locations of the seven X-ray sources with the 2MASS catalog and found that two had infrared counterparts within $1^{\prime \prime}$ of the X-ray sources: CXOGC J174528.6285605 , which is coincident with a previously identified young star in the bright $(\approx 450 \mathrm{mJy}$ at $8.4 \mathrm{GHz})$ extended radio nebula H2 (Yusef-Zadeh \& Morris 1987; Zhao et al. 1993; Figer 1995; Cotera et al. 1999); and the new source CXOGC J174516.1290315 (X174516.1), which is associated with a $2.3 \pm 0.3 \mathrm{mJy}$ unresolved radio source. The chance that one of these radio and X-ray sources randomly would have a 2 MASS counterpart within $1^{\prime \prime}$ is $<15 \%$; if we restrict ourselves to 2MASS sources with $K_{s}<10$, the chance is $<4 \%$. The locations and $J H K_{s}$ magnitudes of the 2MASS counterparts are listed in Table 1, and radio, infrared, and X-ray images of the sources are displayed in Figures 1 and 2.

To determine the nature of the infrared counterparts, we obtained low-resolution $(R=150)$ infrared spectra with SpeX at the Infrared Telescope Facility (IRTF; Fig. 3; Rayner et al. 2003). The spectra were taken on 2004 September 6(UT). The weather was clear, with 0.7 seeing at $J$. The acquisition and reduction of the spectra was carried out as described in Burgasser et al. (2004). To account for background emission, a spectrum was extracted from an adjacent region along the slit that did not contain obvious stellar sources and then subtracted from the source spectrum. The flux values for the spectra were scaled to the 2MASS magnitudes using the filter and optical responses and the atmospheric transmission in Cutri et al. (2003).

Both stars exhibit prominent emission lines (Fig. 3). The strong line emission between 1.8 and $2.0 \mu \mathrm{m}$ cannot be resolved into distinct lines in our low-resolution spectrum. The strongest lines are probably from the Brackett series of $\mathrm{H}$ (Figer et al. 1999), but their equivalent widths cannot be measured because it is not possible to determine the shape of the underlying continuum. We can resolve lines from the continuum at wavelengths longer than $2.0 \mu \mathrm{m}$. In Table 2, we tentatively identify strong lines from $\mathrm{He}$ i and $\mathrm{H} \mathrm{Br} \gamma$ and weaker lines that could be from

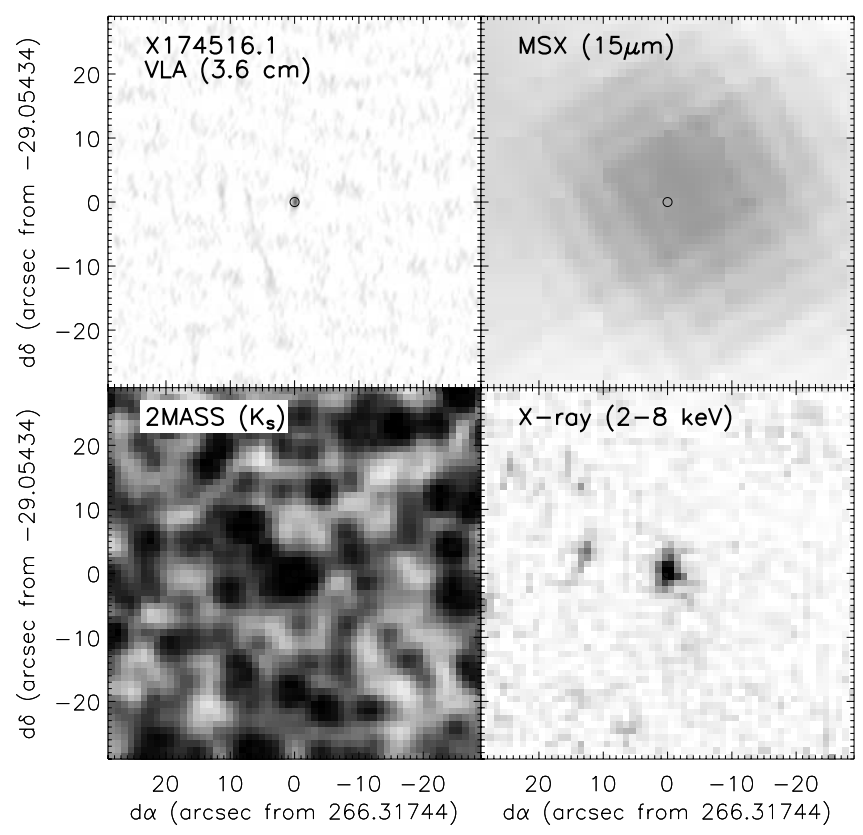

FIG. 1.- Images of CXOGC J174516.1-290315 from the VLA at $3.6 \mathrm{~cm}$, the $M S X$ survey at $15 \mu \mathrm{m}$, the 2MASS survey at $2.2 \mu \mathrm{m}\left(K_{s}\right)$, and Chandra, integrated over the $2-8 \mathrm{keV}$ bandpass. The star is indicated with the circle and is detected as a point source in all of the images. The radio image is displayed on a linear scale with minimum intensity of $0 \mathrm{mJy}$ beam $^{-1}$ and maximum of $3 \mathrm{mJy}^{-1}$ beam $^{-1}$. The $M S X$ and Chandra images are displayed using logarithmic scales. The intensity at $15 \mu \mathrm{m}$ is $33 \mathrm{Jy}$, and the $2-8 \mathrm{keV}$ flux is $5.6 \times 10^{-15}$ ergs $\mathrm{cm}^{-2} \mathrm{~s}^{-1}$. The 2MASS image is displayed using equalized color histograms. The near-infrared sources have a magnitude of $K_{s}=7.9$. 


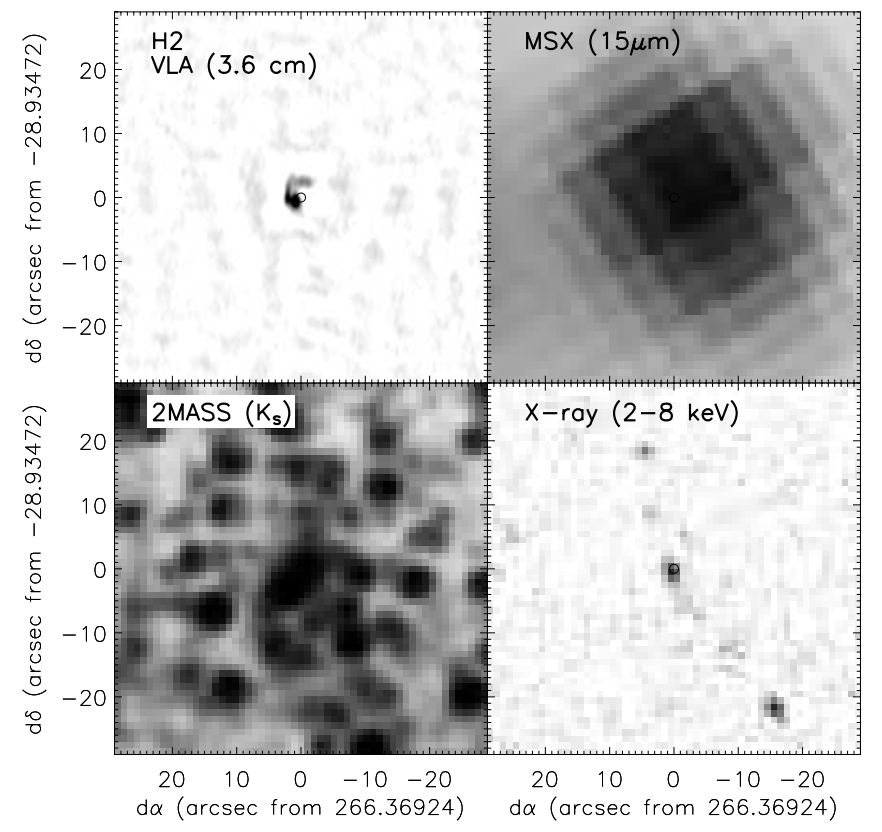

Fig. 2.-Same as Fig. 1, but for H2. The radio image is displayed on a linear scale with minimum intensity of 0 mJy beam ${ }^{-1}$ and maximum of $50 \mathrm{mJy}$ beam $^{-1}$. The remaining images are displayed using the same scalings as Fig. 1. The radio emission is clearly extended, but it appears as a point source in the mid-infrared, infrared, and X-ray. The intensity at $15 \mu \mathrm{m}$ is $220 \mathrm{Jy}$. The nearinfrared sources have a magnitude of $K_{s}=9.2$. The X-ray source has a $2-8 \mathrm{keV}$ flux of $1.7 \times 10^{-15} \mathrm{ergs} \mathrm{cm}^{-2} \mathrm{~s}^{-1}$.

C III, N II, and/or Mg II (see Morris et al. 1996; Figer et al. 1999). We measured the equivalent widths of the lines by modeling the continuum between 2.1 and $2.4 \mu \mathrm{m}$ as a second-order polynomial and the lines as Gaussians. The equivalent widths also are listed in Table 2. The presence of strong $\mathrm{H}$ and $\mathrm{He}$ emission lines indicates that both sources are hot, massive stars.

The radio continuum and infrared-line emission suggested that there could be significant amounts of warm gas and dust surrounding these young stars (e.g., Clark et al. 2003). We searched for this in the mid-infrared, using version 2.3 of the Midcourse Space Experiment (MSX) Point Source Catalog (Egan et al. 2003). X174516.1 and $\mathrm{H} 2$ are the only two sources detected in the X-ray and 2MASS catalogs that also have a counterpart within 1".5 in the $M S X$ catalog. We find that there is a $<15 \%$ chance of finding one random association between the three catalogs. H2 is coincident with MSX6C G259.9845+00.0275 and has fluxes of $33,134,220$, and $490 \mathrm{Jy}$ at $8.3,12.1,14.7$, and $21.3 \mu \mathrm{m}$, respectively. X174516.1 is coincident with MSX6C G359.8590+00.0036 and has fluxes of 2, 14, 33, and 102 Jy at $8.3,12.1,14.7$, and $21.3 \mu \mathrm{m}$, respectively. The fluxes are accurate to $5 \%$, although the angular resolution of $M S X$ was $\approx 20^{\prime \prime}$, so the mid-infrared emission may represent a blend of nearby stars.

As we describe in $\S 3$, the strength of the X-ray emission from these young stars is a bit surprising. Therefore, in order to determine its origin, we determined the $\mathrm{X}$-ray intensities, spectra, and light curves for each star using the acis_extract routine from Tools for X-ray Analysis (TARA) ${ }^{11}$ and CIAO, version 3.2. The techniques are described in Muno et al. (2004), and the dates and exposure times for the observations used are listed in Muno et al. (2005). In brief, for each source we extracted source events from within the $90 \%$ contour of the point-spread func-

\footnotetext{
11 Available at http://www.astro.psu.edu/xray/docs/TARA/.
}
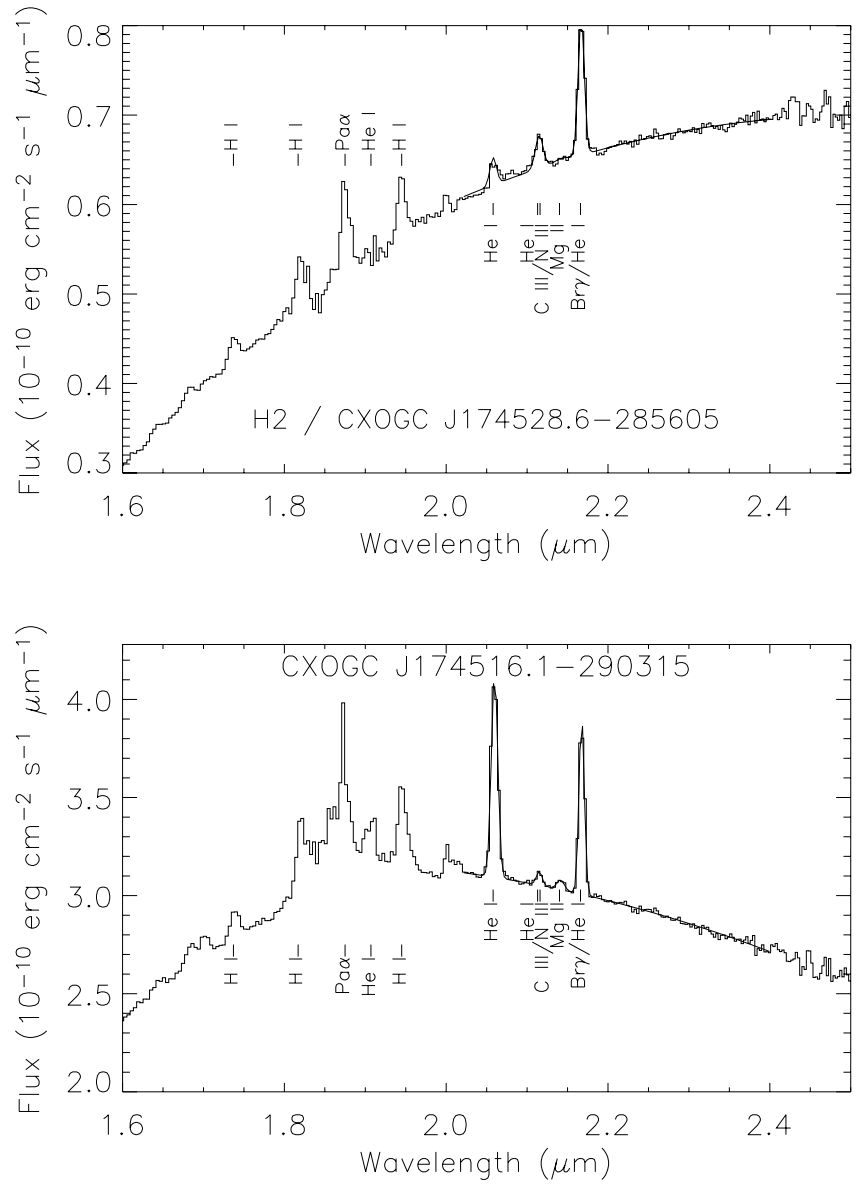

FIG. 3.- Infrared spectrum of the new emission-line star, taken with SpeX on IRTF. The spectrum we obtained extends to shorter wavelengths, but the strong absorption toward the Galactic center cut off the spectrum shortward of $1.7 \mu \mathrm{m}$. The strong $\mathrm{H}, \mathrm{He}$, and $\mathrm{Mg}$ lines are reminiscent of Of and LBV stars in Morris et al. (1996).

tion (PSF) and background events using annular regions that excluded known point sources and discrete filamentary features. We list the net and background X-ray counts from each source in Table 1. These values supersede those in Muno et al. (2003).

We then produced source and background spectra from the event lists, computed the effective area using the CIAO tool mkarf, and obtained the response functions tabulated by Townsley et al. (2002a). The mean fluxes of the sources did not change during

TABLE 2

EMission Lines from Transitional Stars

\begin{tabular}{|c|c|c|c|}
\hline \multirow[b]{2}{*}{ Transition } & \multirow{2}{*}{$\begin{array}{c}\text { CENTROID } \\
(\mu \mathrm{m})\end{array}$} & \multicolumn{2}{|c|}{ EW $(\AA)$} \\
\hline & & $\mathrm{X} 174516.1$ & $\mathrm{H} 2$ \\
\hline He I $2 s^{1} S-2 p^{1} P^{0} \ldots \ldots \ldots \ldots \ldots \ldots$ & 2.058 & $33 \pm 1$ & $5.0 \pm 0.3$ \\
\hline $\begin{array}{l}\text { He I } 2 p^{3} P^{0}-4 s^{3} S \\
\quad+\mathrm{He}_{\text {I }} 3 p^{1} P^{0}-4 s^{1} S \\
\quad+\mathrm{N} \text { III/C } / \mathrm{C} \text { III } 8-7 \ldots \ldots \ldots \ldots \ldots \ldots . . .\end{array}$ & 2.115 & $1.8 \pm 0.6$ & $6.9 \pm 0.6$ \\
\hline $\mathrm{Mg}$ II $5 s^{2} S_{1 / 2}-5 p^{2} P_{1 / 2}^{0} \ldots \ldots \ldots \ldots$ & 2.142 & $2.0 \pm 0.7$ & $<1.9$ \\
\hline $\begin{array}{l}\text { H I } 7-4 \\
\quad+\text { He I } 7 f^{3} F^{0}-4 d^{3} D \\
\quad+\text { He I } 3 p^{1} P^{0}-4 s^{1} S \ldots \ldots \ldots \ldots \ldots . . .\end{array}$ & 2.167 & $25 \pm 1$ & $22.5 \pm 0.3$ \\
\hline
\end{tabular}

NoтE.-The spectra were taken with $R=150$, so at $2 \mu \mathrm{m}$ the resolution is $\approx 0.01 \mu \mathrm{m}$. 
TABLE 3

X-Ray Spectra of Young Stars Near the Galactic Center

\begin{tabular}{|c|c|c|c|c|c|c|}
\hline Infrared ID & $\begin{array}{c}N_{\mathrm{H}} \\
\left(10^{22} \mathrm{~cm}^{-2}\right)\end{array}$ & $\begin{array}{c}k T \\
(\mathrm{keV})\end{array}$ & Norm & $\chi^{2} / \nu$ & $\begin{array}{c}F_{\mathrm{X}} \\
\left(\text { ergs } \mathrm{cm}^{-2} \mathrm{~s}^{-1}\right)\end{array}$ & $\begin{array}{c}L_{\mathrm{X}} \\
\left(\operatorname{ergs~s}^{-1}\right)\end{array}$ \\
\hline $\mathrm{H} 2 \ldots$ & $7.7_{-1.4}^{+1.5}$ & $1.2_{-0.2}^{+0.2}$ & $1.0_{-0.5}^{+1.2} \times 10^{-4}$ & $11 / 11$ & $5.6 \times 10^{-15}$ & $1.4 \times 10^{33}$ \\
\hline 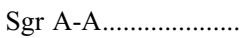 & $6.0^{\mathrm{a}}$ & $>2$ & $4_{-2}^{+3} \times 10^{-6}$ & $5 / 4$ & $1.7 \times 10^{-15}$ & $5 \times 10^{31}$ \\
\hline X174516.1................ & $4.7_{-0.3}^{+0.3}$ & $1.3_{-0.1}^{+0.1}$ & $1.5_{-0.4}^{+0.3} \times 10^{-4}$ & $50 / 37$ & $1.7 \times 10^{-14}$ & $1.9 \times 10^{33}$ \\
\hline
\end{tabular}

Notes.-The fluxes and luminosities are quoted for the $0.5-8.0 \mathrm{keV}$ band. However, as a result of the high extinction toward the Galactic center, most of the observed flux is detected between 2 and $8 \mathrm{keV}$. The luminosities in this band are factors of 2-4 below those in the table.

the first $85 \%$ of the exposures taken through 2003 June (Muno et al. 2004; although see below), so we used summed spectra for each source. We modeled the spectra using XSPEC (ver. 11.3.1; Arnaud 1996), as a thermal plasma (Mewe et al. 1986) absorbed by interstellar gas and dust. The free parameters in this model were the column of interstellar gas $\left(N_{\mathrm{H}, \mathrm{ISM}}\right)$, the plasma temperature $(k T)$ and normalization (proportional to the emission measure $\left.K_{\mathrm{EM}}=\int n_{e} n_{\mathrm{H}} d V\right)$. The optical depth of dust was set to $\tau=0.485 N_{\mathrm{H}} /\left(10^{22} \mathrm{~cm}^{-2}\right)$, and the halo area to 100 times that of the PSF (Baganoff et al. 2003). The best-fit spectral pa-
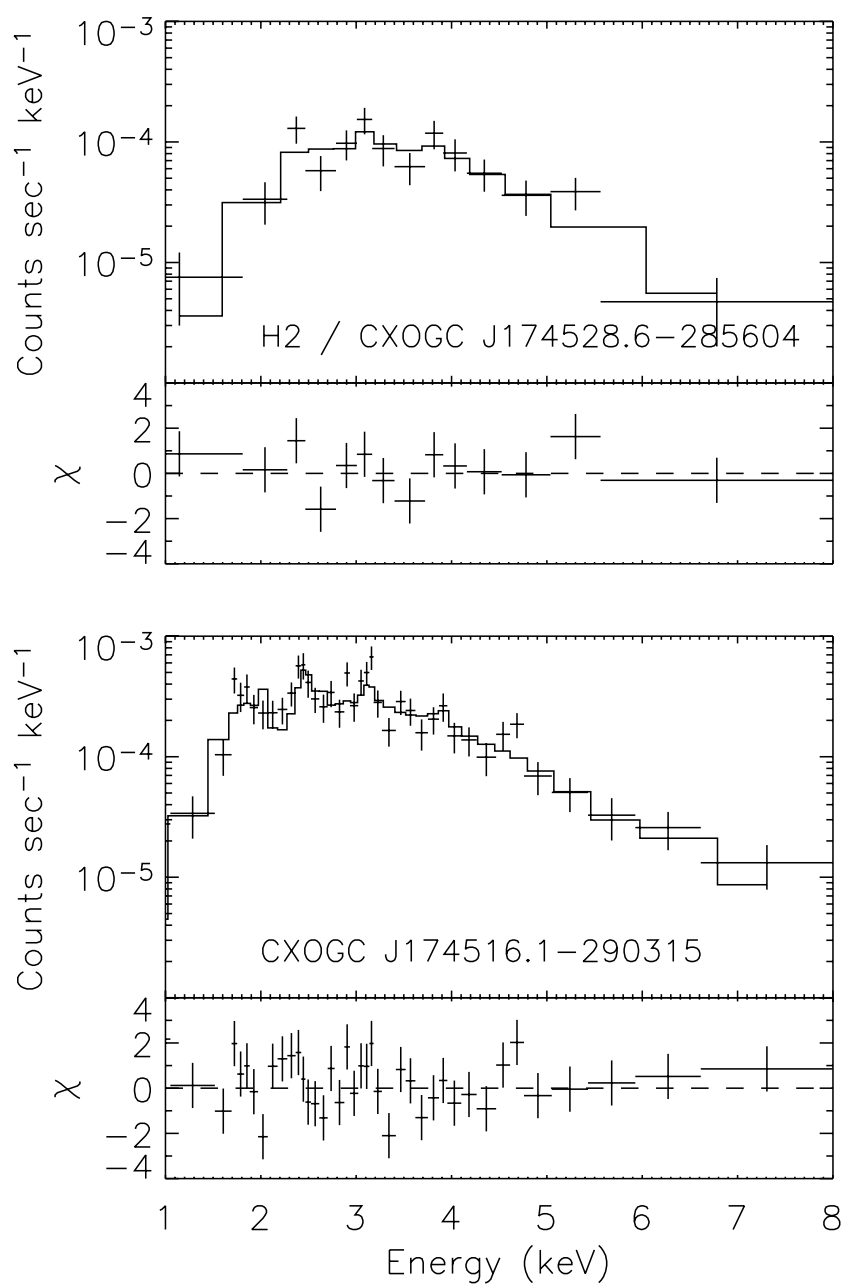

FIG. 4.-X-ray spectra of the two bright X-ray sources, H2 and X174516.1. The top panels display the spectra in detector counts as a function of energy, so the shape of the source spectra are convolved with the detector response. The bottom panels contain the differences between the data and the best-fit thermal plasma models, divided by the Poisson uncertainties on the data points. rameters and derived luminosities for the two detected sources are listed in Table 3, and the spectra are displayed in Figure 4.

Finally, massive stars, particularly those in binaries, are often variable X-ray sources. Therefore, we examined whether X-ray light curves for $\mathrm{H} 2$ and X174516.1 were consistent with a constant mean flux using Kolmogorov-Smirnov (K-S) and $\chi^{2}$ tests. The photon flux from X174516.1 increased by a factor of 3, from $(3.1 \pm 0.2) \times 10^{-6}$ photons $\mathrm{cm}^{-2} \mathrm{~s}^{-1}$ between 1999 and 2003 to $(7.8 \pm 0.7) \times 10^{-6}$ photons $\mathrm{cm}^{-2} \mathrm{~s}^{-1}$ during 2004 July. The probability that this increase in flux resulted from a constant flux was $<2 \times 10^{-7}$ under a $\chi^{2}$ test. We find no evidence that the hardness of the spectrum varied during the outburst. We defined a hardness ratio $(h-s) /(h+s)$, using $s$ as the number of counts in the $0.5-3.0 \mathrm{keV}$ band and $h$ as the counts in the 3.0$8.0 \mathrm{keV}$ band. The average hardness from 1999 through 2003 was $0.02 \pm 0.06$, while that during 2004 July was $-0.01 \pm 0.09$, so there is no evidence for spectral variations coincident with the increase in flux. On shorter timescales, there appears to have been a flare near or before the start of the observation on 2004 July 6 , which decayed with a timescale of $\approx 1 \mathrm{hr}$. During that observation, the chance that the data were produced by a constant flux was $<0.3 \%$ according to a K-S test.

The X-ray light curve from $\mathrm{H} 2$ has a $\approx 30 \%$ chance of being produced by a constant mean flux under both tests. However, because the count rate from $\mathrm{H} 2$ is lower than that from X174516.1, we cannot exclude the hypothesis that a flux variation with a factor of $\lesssim 3$ amplitude occurred between 1999 and 2004 .

\subsection{X-Ray Counterparts to Known Emission-Line Stars}

After realizing that $\mathrm{H} 2$ had been previously identified as a young star, we returned to previous searches for such stars to determine if others were also strong X-ray sources. For the purposes of this paper, we are interested in relatively isolated stars, ${ }^{12}$ so we searched for X-ray counterparts to the four emission-line stars (besides H2) in Cotera et al. (1999). We first compared the locations and JHK magnitudes of the Cotera et al. (1999) stars to the 2MASS catalog in order to refine their locations. We found that the published positions of the stars disagreed with their 2 MASS counterparts by $1^{\prime \prime}-3^{\prime \prime}$. Therefore, we report updated positions and the $J H K_{s}$ magnitudes of the emission-line stars in Table 1. Using the updated positions, we found that one more of the remaining four emission-line stars has an X-ray counterpart in our Chandra image within the 0.5 uncertainty of the X-ray catalog: the WN 6 star Sgr A-A. ${ }^{13}$ This star was not detected in our radio and X-ray comparison $(\S 2.1)$, because the infrared

\footnotetext{
12 The X-ray properties of the stars in the central parsec will be reported in a future publication.

13 We note that the inaccurate positions in Cotera et al. (1999) led us to report initially that none had counterparts (Muno et al. 2004).
} 


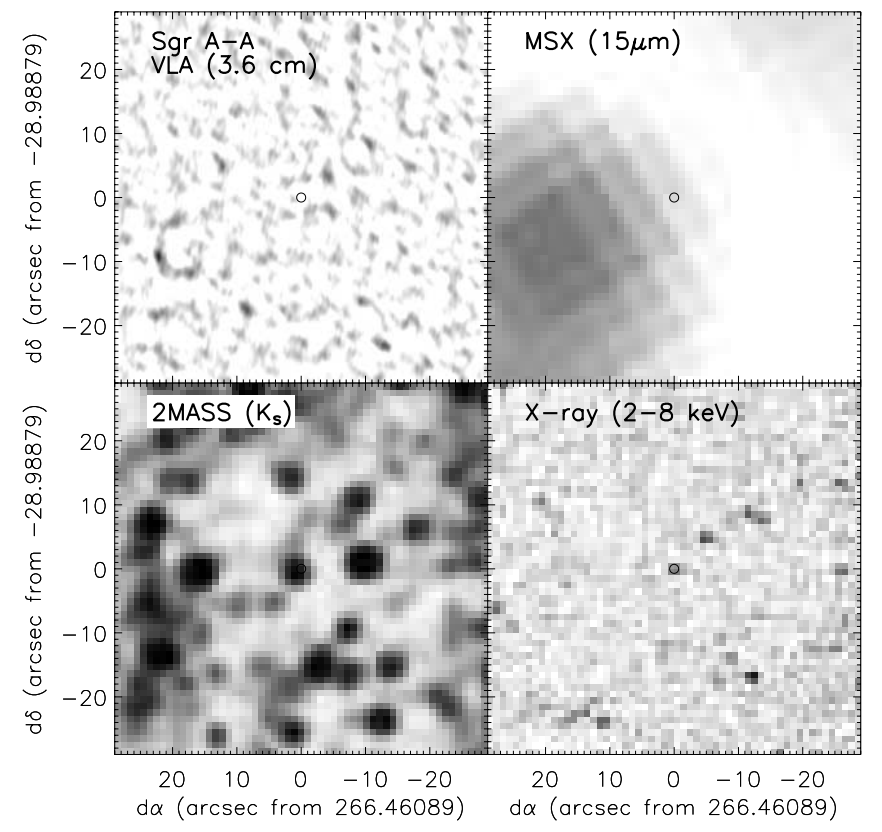

Fig. 5.-Same as Fig. 1, but for the W-R star near Sgr A-A. The radio image is displayed on a linear scale with minimum intensity of $0 \mathrm{mJy}^{-1}$ beam ${ }^{-1}$ and maximum of $50 \mathrm{mJy} \mathrm{beam}^{-1}$. The remaining images are displayed using the same scalings as Fig. 1. The W-R star is visible as a point source in the infrared and X-rays. The upper limit to the flux density of a pointlike radio source coincident with the W-R star is $<2.4 \mathrm{mJy}$. The $\mathrm{H}$ I r region Sgr A-A can be seen in the mid-infrared, located $20^{\prime \prime}$ east and $10^{\prime \prime}$ south of the W-R star. The VLA observations have resolved out the diffuse radio emission.

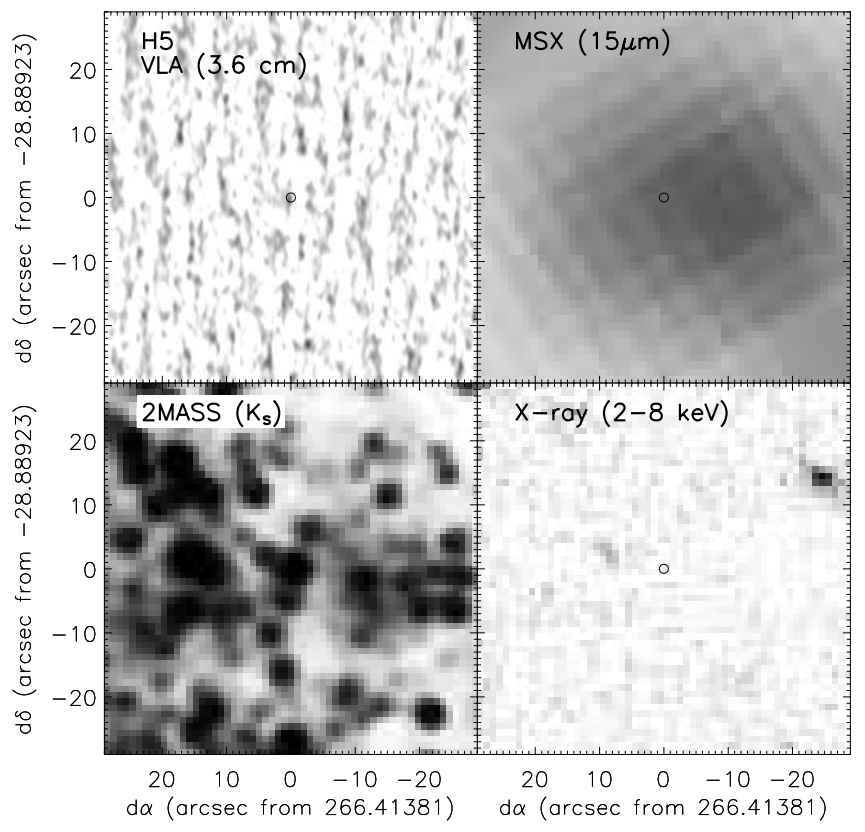

Fig. 7. - Same as Fig. 1, but for the star associated with the radio nebula H5. The radio image is displayed on a linear scale with minimum intensity of $0 \mathrm{mJy}$ beam $^{-1}$ and maximum of $3 \mathrm{mJy}_{\text {beam }}{ }^{-1}$. The remaining images are displayed using the same scalings as Fig. 1. The star is only visible in the infrared image; it is not detected in X-rays. The VLA observations have resolved out the emission from the $\mathrm{H}$ II region, and the upper limit to the flux density of a pointlike radio source is $<1.6 \mathrm{mJy}$ There is extended mid-infrared emission from the nebula.

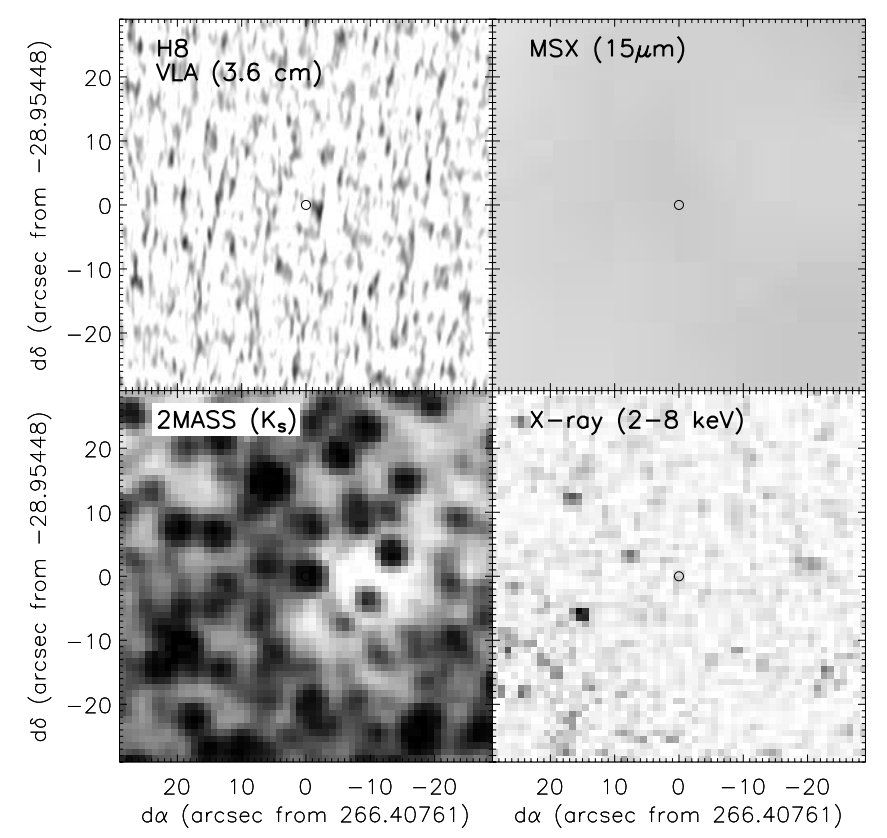

FIG. 6.- Same as Fig. 1, but for the star associated with the radio nebula H8. The radio image is displayed on a linear scale with minimum intensity of $0 \mathrm{mJy}$ beam $^{-1}$ and maximum of $3 \mathrm{mJy}_{\text {beam }}{ }^{-1}$. The remaining images are displayed using the same scalings as Fig. 1. The star is only visible in the infrared image; it is not detected in X-rays. The VLA observations have resolved out the emission from the $\mathrm{H}$ II region, and the upper limit to the flux density of a pointlike radio source is $<1.2 \mathrm{mJy}$. The mid-infrared emission exhibits a diffuse background that is not resolved into discrete features.

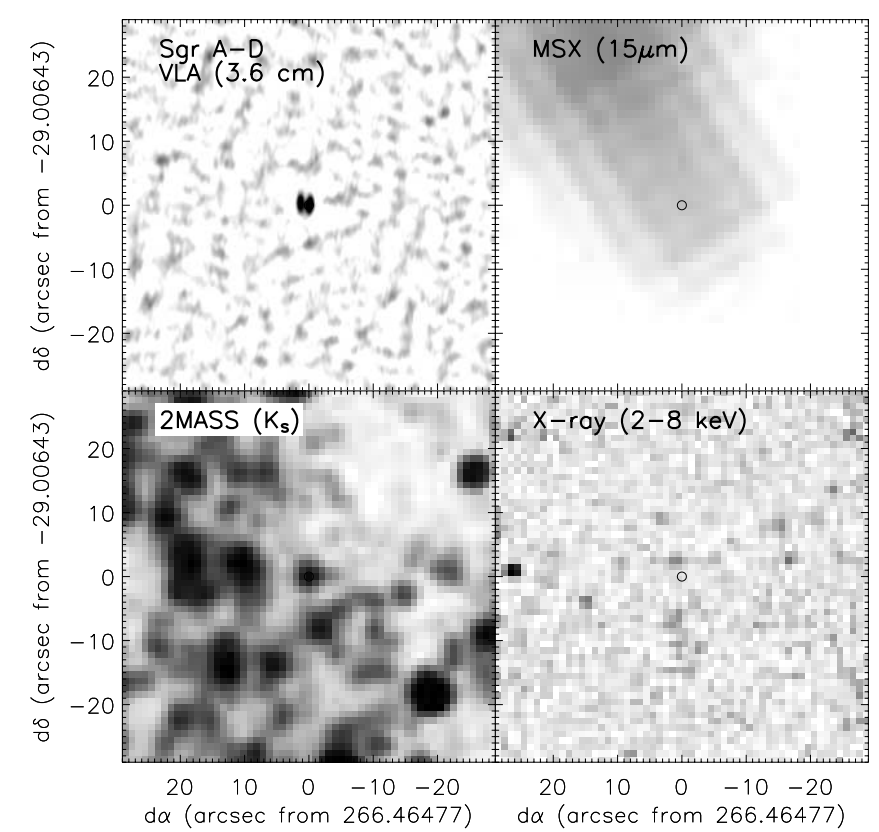

FIG. 8.-Same as Fig. 1, but for the star associated with the radio nebula Sgr A-D. The radio image is displayed on a linear scale with minimum intensity of $0 \mathrm{mJy}$ beam $^{-1}$ and maximum of $50 \mathrm{mJy}^{\mathrm{beam}}{ }^{-1}$. The remaining images are displayed using the same scalings as Fig. 1 . The star is visible in the infrared image, and is coincident with two pointlike radio features with fluxes of $22 \mathrm{mJy}$. Most of the radio emission from the $\mathrm{H}$ II region is resolved out, but it can be seen in the mid-infrared image. The star is not detected in X-rays. 
TABLE 4

X-Ray and Bolometric Luminosities of Transitional Massive Stars

\begin{tabular}{|c|c|c|c|c|c|}
\hline Star & Type & $\begin{array}{c}L_{\mathrm{bol}} \\
\left(\log \left[\operatorname{ergs~s}^{-1}\right]\right)\end{array}$ & $\begin{array}{c}L_{\mathrm{X}} \\
\left(\log \left[\operatorname{ergs~s}^{-1}\right]\right)\end{array}$ & $\log L_{\mathrm{X}} / L_{\mathrm{bol}}$ & References \\
\hline X174516.1............. & $\ldots$ & 39.7 & 33.3 & -6.4 & 1 \\
\hline 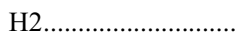 & $\ldots$ & 39.5 & 33.1 & -6.4 & 1 \\
\hline 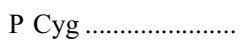 & LBV & 39.5 & $<31.0^{\mathrm{a}}$ & $<-8.5$ & 2,3 \\
\hline$\eta$ Car & LBV & 40.1 & 34.8 & -5.3 & 2,4 \\
\hline Pistol................................... & LBVc & 40.2 & $<32.0^{\mathrm{b}}$ & $<-8.2$ & 1,5 \\
\hline HD 108 & $\mathrm{O} 6 \mathrm{f}$ & 39.2 & 33.0 & -6.2 & 6 \\
\hline 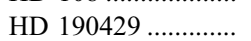 & 04.5 If $^{+}$ & 39.7 & $31.1^{\mathrm{a}, \mathrm{c}}$ & -8.6 & $7,8,9$ \\
\hline HD $152408 \ldots \ldots \ldots \ldots . . .$. & O8 Iafpe & 39.4 & $<31.7^{\mathrm{a}}$ & $<-7.7$ & 7,10 \\
\hline HD $151804 \ldots \ldots \ldots \ldots . . .$. & O8 Iaf & 39.7 & $31.9^{\mathrm{a}, \mathrm{c}}$ & -7.8 & $7,11,12$ \\
\hline
\end{tabular}

Notes.-The bolometric luminosities for the two sources in this work are estimated by assuming values for the extinction and the bolometric correction for each star, as described in the text. The X-ray luminosities are extrapolated from the observed values into the $0.5-8.0 \mathrm{keV}$ band, except where indicated. The $0.5-8.0 \mathrm{keV}$ luminosities of massive stars are usually dominated by $k T \approx 0.5 \mathrm{keV}$ plasma that would be undetectable through the absorption toward the Galactic center; at most $10 \%$ of $L_{\mathrm{X}}$ should be produced by hotter $1 \mathrm{keV}$ plasma. Therefore, both luminosity values are uncertain by roughly an order of magnitude.

${ }^{\text {a }}$ The luminosities are reported in the ROSAT $0.1-2.0 \mathrm{keV}$ band for these sources. For direct comparison with the other sources, one would want to use the $0.5-8.0 \mathrm{keV}$ luminosity, which will in general be a factor of $\sim 10$ smaller.

${ }^{\mathrm{b}}$ We derived an upper limit to the X-ray luminosity for the Pistol star using archival Chandra data, in the same manner as we did for the other stars in our survey.

${ }^{\mathrm{c}}$ We assumed that 0.01 counts s $\mathrm{s}^{-1}$ in the ROSAT PSPC equals $1 \times 10^{-13} \mathrm{ergs} \mathrm{cm}^{-2} \mathrm{~s}^{-1}$, which is appropriate for a $0.5 \mathrm{keV}$ thermal plasma spectrum absorbed by $5 \times 10^{20} \mathrm{~cm}^{-2}$ of $\mathrm{H}$ (see, e.g., Berghöfer et al. 1996). The true correction can vary by a factor of 4 for reasonable choices of the assumed absorption and temperature.

REFERENCES.- (1) this work; (2) Humphreys \& Davidson 1994; (3) Berghöfer \& Wendker 2000; (4) Seward et al. 2001; (5) Figer et al. 1999; (6) Nazé et al. 2004; (7) Morris et al. 1996; (8) Scuderi et al. 1998; (9) Voges et al. 1999; (10) Berghöfer et al. 1996; (11) ROSAT PSPC Pointed Observations catalog; (12) Lamers \& Leitherer 1993.

and $\mathrm{X}$-ray sources are significantly offset from the nearby radio $\mathrm{H}$ II region. We display images of Sgr A-A in Figure 5.

We analyzed the X-ray emission from each of the four stars in the same manner as discussed in $\S 2.2$ and list their properties in Table 3. For the three nondetections from Cotera et al. (1999), we computed limits on their count rates by extracting events from the 2MASS positions ( Table 1). The limits on their luminosities for a $1 \mathrm{keV}$ thermal plasma spectrum are $1 \times 10^{32} \mathrm{ergs} \mathrm{s}^{-1}$, and for a $0.5 \mathrm{keV}$ plasma $2 \times 10^{33} \mathrm{ergs} \mathrm{s}^{-1}$. Images of the stars not detected in X-rays are displayed in Figures 6, 7, and 8.

\section{DISCUSSION}

We have searched for X-ray emission from young stars located between 3-23 pc in projection from Sgr A*, and we have detected two of five known isolated, young stars (Cotera et al. 1999) and identified one new example. The stars that we detected in our Chandra image are exceptional. The star associated with the Sgr A-A H II region is the only Wolf-Rayet star (spectral type WN 6) in the sample of Cotera et al. (1999). Nitrogen-rich Wolf-Rayet (WN) stars produce very fast winds $\left(v \gtrsim 1000 \mathrm{~km} \mathrm{~s}^{-1}\right)$, which produce strong X-ray-emitting shocks. About $75 \%$ of WN stars have $L_{\mathrm{X}}>5 \times 10^{31} \mathrm{ergs} \mathrm{s}^{-1}$ (Wessolowski 1996), so the detection of the Sgr A-A WN 6 star is not surprising (Table 3).

The two most luminous X-ray sources, X174516.1 and H2, are the brightest infrared sources in Table 1. We compared their spectra (Fig. 3) to those of the Pistol star (Figer et al. 1998) and to the atlases of Morris et al. (1996) and Hanson et al. (1996). Our low-resolution spectra cannot be used to classify stars with the detail achievable with high-resolution optical spectra. However, the line emission from $\mathrm{Br} \gamma, \mathrm{He}$ I, and $\mathrm{Mg}$ II in Figure 3 resembles that from the Pistol star and other $\mathrm{B}[\mathrm{e}]$ supergiants, Of stars, and luminous blue variables (LBVs). All of these spectral types represent massive stars that have evolved off of the main sequence but not yet reached the Wolf-Rayet phase, so the simi- larities are understandable. For $\mathrm{H} 2$, our results match the conclusions of Figer (1995) and Cotera et al. (1999).

Our suggestion that these objects are B[e], Of, or LBV stars suggests that they are unusually luminous. To confirm this, we estimate the intrinsic colors and luminosities of X174516.1 and $\mathrm{H} 2$ as follows. First, we assume that they lie near the Galactic center $(D=8 \mathrm{kpc})$, because (1) the interstellar absorption columns that we measure from their X-ray spectra (Table 3 ) are quite similar to the value toward $\operatorname{Sgr~A}^{*}\left(N_{\mathrm{H}}=6 \times 10^{22} \mathrm{~cm}^{-2}\right)$, and (2) if we ignore the heavily absorbed half of the Galaxy more distant than $10 \mathrm{kpc}, \sim 80 \%$ of the Galactic stellar mass along this line of sight is located within $\pm 200 \mathrm{pc}$ of the Galactic center (Launhardt et al. 2002). The distance modulus is therefore 14.5. Second, we estimate the reddening using the relationship $N_{\mathrm{H}} / A_{V}=1.79 \times 10^{21} \mathrm{~cm}^{-2}$ from Predehl \& Schmitt (1995) and the extinction law from Moneti et al. (2001). For X174516.1, taking $N_{\mathrm{H}}=(4.4 \pm 0.3) \times 10^{22} \mathrm{~cm}^{-2}$, we find $A_{H}=4.6 \pm 0.3$, and $A_{K}=2.9 \pm 0.2$. Its intrinsic color is $\left(H-K_{S}\right)_{0}=-0.4 \pm 0.1$, and absolute $M_{K}=-9.5 \pm 0.2$. For $\mathrm{H} 2, N_{\mathrm{H}}=(7.7 \pm 1.4) \times 10^{22} \mathrm{~cm}^{-2}$, we find $A_{H}=7.5 \pm 1.4$, and $A_{K}=4.8 \pm 0.9$. Its intrinsic color is $\left(H-K_{s}\right)_{0}=-0.6 \pm$ 0.5 and $M_{K}=-10.1 \pm 0.9$. In both cases, the colors are within $1 \sigma$ of $(H-K)_{0}=-0.3 \pm 0.1$, as expected for $\mathrm{O}$ supergiant stars (Wegner 1994). However, the colors are inconsistent with the $(H-K)_{0} \gtrsim 0.8$ infrared excesses seen from $\mathrm{B}[\mathrm{e}]$ stars in the Magellanic Clouds (Zickgraf et al. 1986). Therefore, we tentatively classify X174516.1 and H2 as either Of or candidate LBV stars.

We estimate the bolometric luminosities of these stars by assuming that they have temperatures comparable to those of the Pistol star and other hot, massive stars in transition, $T \gtrsim$ $1.5 \times 10^{4} \mathrm{~K}$ (Morris et al. 1996; Figer et al. 1998; Clark et al. 2003). The bolometric corrections to the absolute $K_{S}$ magnitudes will then be given by $\mathrm{BC}_{K}=-7.1 \log T+28.8$ (Blum et al. 1995), so that $\mathrm{BC}_{K} \lesssim-0.9$. Therefore, we find upper limits 
on the bolometric magnitudes for X174516.1 of $M_{\mathrm{bol}} \lesssim-10.2$, and for $\mathrm{H} 2$ of $M_{\text {bol }} \lesssim 10.1$. We converted these to lower limits on the luminosities (listed in Table 4), and find for X174516.1 that $L_{\text {bol }}>10^{6.1} L_{\odot}$, and for $\mathrm{H} 2$ that $L_{\text {bol }}>10^{5.9} L_{\odot}$. Their true luminosities may be significantly higher. For example, assuming $T=30,000 \mathrm{~K}$, as would be appropriate for an Of star, the bolometric luminosity would be a factor of 7 higher.

\subsection{Radio, Mid-Infrared, and X-Ray Emission}

Although X174516.1 and H2 are very luminous stars, it is nonetheless surprising that they are so bright in the radio, midinfrared, and X-rays. Free-free emission in the winds of massive, evolving stars should make them millijansky radio sources from the distance to the Galactic center (e.g., Scuderi et al. 1998; Duncan \& White 2002). The pointlike radio source consistent with X174516.1 is consistent with a stellar wind. We can estimate the required mass-loss rate by inverting equation (4) in Scuderi et al. (1998):

$$
\dot{M}=2 \times 10^{-7}\left(S_{\nu} d_{\mathrm{kpc}}^{2} \nu_{10 \mathrm{GHz}}^{-0.6} T_{4}^{0.1}\right)^{3 / 4} \frac{\mu_{e} v_{\infty}}{10^{2} \mathrm{~km} \mathrm{~s}^{-1}} M_{\odot} \mathrm{yr}^{-1}
$$

If we assume the electron temperature in units of $10^{4} \mathrm{~K}$ is $T_{4}=1$, the mean atomic weight per free electron is $\mu_{e}=1.3$, and the terminal velocity of the wind is $v_{\infty}=200 \mathrm{~km} \mathrm{~s}^{-1}$, as appropriate for an LBV (e.g., P Cyg in Scuderi et al. 1998), then $S_{8.4 \mathrm{GHz}}=2.3 \mathrm{mJy}$ implies $\dot{M}=2 \times 10^{-5} M_{\odot} \mathrm{yr}^{-1}$. This is similar to the mass-loss rates observed for LBVs (see also, e.g., Clark et al. 2003). A higher velocity of $v_{\infty} \approx 1000 \mathrm{~km} \mathrm{~s}^{-1}$, as appropriate for an Of star, would imply a mass-loss rate $>10^{-4} M_{\odot} \mathrm{yr}^{-1}$. This rate is much higher than is typically seen in Of stars (Scuderi et al. 1998). However, since we have not measured the radio spectral index of X174516.1, it is possible that most of the flux is nonthermal, and the above mass-loss rates are upper limits. To determine the nature of the radio emission from X174516.1, either the radio spectral index must be measured or the mass-loss rate must be obtained from a highresolution infrared spectrum.

The radio flux of the extended nebula around $\mathrm{H} 2$ is orders of magnitude too bright to be produced by its stellar wind. Zhao et al. (1993) model it as an unusually dense $\left(n_{e} \sim 10^{3} \mathrm{~cm}^{-3}\right) \mathrm{H} \mathrm{II}$ region illuminated by a Lyman continuum flux equivalent to that of an $\mathrm{O} 7$ zero-age main-sequence star.

The detection of both stars in the MSX mid-infrared survey demonstrates that they are surrounded by warm gas and dust. Egan et al. (2002) and Clark et al. (2003) have identified dusty ring nebulae around several known and candidate LBV stars and hypothesize that they were produced by past episodes of rapid mass loss. Surprisingly, the mid-infrared counterparts to our Galactic center stars are $\gtrsim 10$ and 100 times more luminous at 14.7 and $21.3 \mu \mathrm{m}$, respectively, than the nebulae around the other candidate LBVs. Therefore, we suspect that the midinfrared flux near X174516.1 and H2 either represents interstellar dust or is the unresolved emission from several massive, dusty stars. Observations with the Spitzer Space Telescope should resolve this issue.

The detection of X174516.1 and H2 with Chandra is particularly intriguing because Of and LBV stars are not always bright X-ray sources. In Table 4 , we tabulate the values of $L_{\mathrm{X}} / L_{\mathrm{bol}}$ for several of the massive stars listed in Morris et al. (1996) and Figer et al. (1999).

The measurements of $L_{\mathrm{X}}$ were taken with instruments sensitive to different energy ranges, so some caution should be used in comparing the results, as described in the notes to the table. In general, we have extrapolated the fluxes using conservative assumptions that serve to lessen the dispersion in $L_{X} / L_{\text {bol }}$. Therefore, we expect that the range in these values is not caused by selection effects but represents intrinsic differences in the efficiency of X-ray production in these sources. We find that there is at least a factor of 3000 range in the values and that the scatter is not correlated with spectral type. The LBV $\eta$ Car and the $\mathrm{O} 6 \mathrm{f}$ star HD 108 lie near the X-ray-bright end of the distribution with $L_{\mathrm{X}} / L_{\text {bol }}=-5.3$ and -6.2 , respectively. The LBV P Cyg and the O4.5 If star HD 190429 lie at the faint end, with $L_{\mathrm{X}} / L_{\mathrm{bol}}<-8.5$.

$\mathrm{X} 174516.1$ and $\mathrm{H} 2$ lie at the high end of the scatter in $L_{\mathrm{X}} / L_{\mathrm{bol}}$ in Table 4, which raises the question of why they are so bright in $\mathrm{X}$-rays. There are several possible explanations. One is that the $\mathrm{X}$-ray-emitting shocks form at varying optical depths within the winds of massive stars, so that in some cases the X-ray emission is absorbed before it can reach the observer. This has been proposed to explain why carbon-type W-R stars are faint X-ray sources, whereas nitrogen-type W-R stars are bright in X-rays (Oskinova et al. 2003). However, changes in the optical depth of the wind from X174516.1 are unlikely to explain the X-ray flare observed in 2004 July ( $§ 2.1$ ). Alternatively, the X-rays from X174516.1 and H2 could be produced when their winds collide with those of fainter companions. For instance, it has been proposed that the high X-ray luminosity of $\eta$ Car is produced because it is a binary and one of the components has a fast, $v \sim 1000 \mathrm{~km} \mathrm{~s}^{-1}$, wind that collides with the slow, dense wind of its companion (Seward et al. 2001). Finally, these stars could have black hole or neutron star companions that produce $\mathrm{X}$-rays as they accrete from the wind of the supergiant. This would make X174516.1 and H2 analogous to faint high-mass $\mathrm{X}$-ray binaries such as 1A $0535+26$ and X Per, which have similar X-ray spectra and luminosities (Di Salvo et al. 1998; Orlandini et al. 2004) and exhibit prominent $\mathrm{He} \mathrm{I}$ and $\mathrm{Br} \gamma$ emission in their spectra (Hanson et al. 1996). Both colliding-wind binaries and accreting compact objects exhibit X-ray flares similar to that seen from X174516.1. Further high-resolution spectroscopic observations will allow us to model the winds from X174516.1 and $\mathrm{H} 2$ and to search for evidence for binary companions.

Given the scatter in $L_{\mathrm{X}} / L_{\text {bol }}$ in Table 4 , it is not surprising that we did not detect $\mathrm{X}$-ray emission from the remaining three stars from Cotera et al. (1999). The spectra from Cotera et al. (1999) suggest that these stars are all in similar evolutionary states to $\mathrm{X} 174516.1$ and H2. If we make similar assumptions to compute their $L_{\mathrm{bol}}$, we find that the remaining stars are only a factor of a few less luminous than X174516.1 and H2. However, if we assume that they are producing X-rays with characteristic temperatures of $\lesssim 0.5 \mathrm{keV}$, then most of the X-ray emission would be undetectable through the absorption toward the Galactic center, and from the upper limits in $\S 2.1$ we find $\log \left(L_{X} / L_{\text {bol }}\right)<-6$. These limits are not particularly interesting in the context of $\mathrm{X}$-ray emission from massive stars. Nonetheless, we can confidently state that these stars are at least an order of magnitude fainter than X174516.1 and $\mathrm{H} 2$ in the observable $2-8 \mathrm{keV}$ band (Table 1), which highlights the oddity of the latter two sources.

\subsection{Implications for Star Formation}

Hot, post-main-sequence Wolf-Rayet, B[e], Of, and LBV stars similar to those in Table 1 are thought to have initial masses of $M \gtrsim 35 M_{\odot}$ (Zickgraf et al. 1986; Figer et al. 1998) and ages of only a few million years. As a result, such stars are excellent tracers of recent star formation. 
Surprisingly, unlike the Pistol star and the massive stars in the central parsec around Sgr A* (Figer et al. 1998; Paumard et al. 2003), none of the stars in Table 1 is associated with any known young star cluster. However, clusters less massive than the Arches and Quintuplet would be difficult to identify against the dense background of stars in the Galactic center using previous wide-field infrared surveys like 2MASS (Portegies-Zwart et al. 2002). Therefore, it is important to carry out photometric and narrow-line surveys to search for other young stars in the vicinity of $\mathrm{H} 2$ and X174516.1, to establish whether they are in fact isolated.

If they are isolated, then there are two possible explanations. First, it has been suggested that the young stars in the central parsec are the remnants of a cluster that was formed $\sim 10 \mathrm{pc}$ from Sgr A* and settled into the Galactic center (Gerhard 2001); stars like H2 and X174516.1 could have been stripped from this hypothetical cluster. If this is the case, then hundreds of other young stars still await discovery in the central $10 \mathrm{pc}$ around Sgr $\mathrm{A}^{*}$ (e.g., Portegies Zwart et al. 2003; Kim \& Morris 2003). Alternatively, these massive, young stars may well have formed in isolation, as has been proposed by Cotera et al. (1999). This hypothesis would imply that stars form in associations that have masses ranging from 100 to $10^{4} M_{\odot}$. Such a conclusion would be sur- prising, since the high turbulent velocities of molecular clouds, the large tidal forces produced by Sgr $\mathrm{A}^{*}$ and the surrounding stellar cusp, and possibly milligauss magnetic fields concentrated near the Galactic center by infalling material should collude to make the Jeans mass closer to the upper end of this range (Morris 1993).

We thank D. Figer, A. Cotera, E. Feigelson, L. Townsley, and J. S. Clark for very helpful discussions about the natures of these young stars and S. Eikenberry for helpful advice about identifying counterparts to the X-ray sources. M. P. M. was supported through a Hubble Fellowship grant (program HSTHF-01164.01-A) from the Space Telescope Science Institute, which is operated by the Association of Universities for Research in Astronomy, Inc., under NASA contract NAS5-26555. A. J. B. acknowledges support from NASA through the Spitzer Fellowship Program. W. N. B. was supported by NSF CAREER Award 9983783. This publication makes use of data products from the Two Micron All-Sky Survey, which is a joint project of the University of Massachusetts and the Infrared Processing and Analysis Center, California Institute of Technology, funded by NASA and the NSF.
Arnaud, K. A. 1996, in ASP Conf. Ser. 101, Astronomical Data Analysis Software and Systems V, ed. G. H. Jacoby \& J. Barnes (San Francisco: ASP), 17 Baganoff, F. K., et al. 2003, ApJ, 591, 891

Berghöfer, T. W., Schmitt, J. H. M. M., \& Cassinelli, J. P. 1996, A\&AS, 118, 481

Berghöfer, T. W., \& Wendker, H. J. 2000, Astron. Nachr., 321, 249

Blum, R. D., DePoy, D. L., \& Sellgren, K. 1995, ApJ, 441, 603

Burgasser, A. J., McElwain, M. W., Kirkpatrick, J. D., Cruz, K. L., Tinney, C. G., \& Reid, I. N. 2004, AJ, 127, 2856

Clark, J. S., Egan, M. P., Crowther, P. A., Mizuno, D. R., Larionov, V. M., \& Arkharov, A. 2003, A\&A, 412, 185

Cotera, A. S., Simpson, J. P., Erickson, E. F., Colgan, S. W. J., Burton, M. G., \& Allen, D. A. 1999, ApJ, 510, 747

Cutri, R. M., et al. 2003, Explanatory Supplement to the 2MASS All Sky Data Release (Pasadena: JPL), http://www.ipac.caltech.edu/2mass/releases/allsky/ doc/explsup.html

de Pree, C. G., Goss, W. M., \& Gaume, R. A. 1998, ApJ, 500, 847

Di Salvo, T., Burderi, L., Robba, N. R., \& Guainazzi, M. 1998, ApJ, 509, 897

Duncan, R. A., \& White, S. M. 2002, MNRAS, 330, 63

Egan, M. P., Clark, J. S., Mizuno, D. R., Carey, S. J., Steele, I. A., \& Price, S. D. 2002, ApJ, 572, 288

Egan, M. P., et al. 2003, Air Force Research Laboratory Technical Report AFRL-VS-TR-2003-1589 (Washington: GPO)

Figer, D. F. 1995, Ph.D. thesis, UCLA

Figer, D. F., Kim, S. S., Morris, M., Serabyn, E., Rich, R. M., \& McLean, I. A. 1999, ApJ, 525, 750

Figer, D. F., Najarro, F., Morris, M., McLean, I. S., Geballe, T. R., Ghez, A. M., \& Langer, N. 1998, ApJ, 506, 384

Figer, D. F., et al. 2004, ApJ, 601, 319

Gerhard, O. 2001, ApJ, 546, L39

Hanson, M. M., Conti, P. S., \& Reike, M. J. 1996, ApJS, 107, 281

Humphreys, R. M., \& Davidson, K. 1994, PASP, 106, 1025

Kim, S. S., \& Morris, M. 2003, ApJ, 597, 312

Krabbe, A., et al. 1995, ApJ, 447, L95

Lamers, H. J. G. L. M., \& Leitherer, C. 1993, ApJ, 412, 771
Launhardt, R., Zylka, R., \& Mezger, P. G. 2002, A\&A, 384, 112

Mewe, R., Lemen, J. R., \& van den Oord, G. H. J. 1986, A\&AS, 65, 511

Moneti, A., Stolovy, S., Blommaert, J. A. D. L., Figer, D. F., \& Najarro, F. 2001, A\&A, 366, 106

Morris, M. 1993, ApJ, 408, 496

Morris, P. W., Eenens, P. R. J., Hanson, M. M., Conti, P. S., \& Blum, R. D. 1996, ApJ, 470, 597

Muno, M. P., Lu, J. R., Baganoff, F. K., Brandt, W. N., Garmire, G. P., Ghez, A. M., Hornstein, S. D., \& Morris, M. R. 2005, ApJ, 633, 228

Muno, M. P., et al. 2003, ApJ, 589, 225 2004, ApJ, 613, 1179

Nazé, Y., Rauw, G., Vreux, J.-M., \& De Becker, M. 2004, A\&A, 417, 667

Orlandini, M., et al. 2004, Nucl. Phys. B Suppl., 132, 476

Oskinova, L. M., Ignace, R., Hamann, W.-R., Pollock, A. M. T., \& Brown, J. C. 2003, A\&A, 402, 755

Paumard, T., Maillard, J.-P., \& Stolovy, S. 2003, Astron. Nachr., 324, 303

Portegies-Zwart, S. F., McMillan, S. L. W., \& Gerhard, O. 2003, ApJ, 593, 352

Portegies-Zwart, S. F., et al. 2002, ApJ, 565, 265

Predehl, P., \& Schmitt, J. H. M. M. 1995, A\&A, 293, 889

Rayner, J. T., Toomey, D. W., Onaka, P. M., Denault, A. J., Stahlberger, W. E., Vacca, W. D., Cushing, M. C., \& Wang, S. 2003, PASP, 155, 362

Scuderi, S., Panagia, N., Stanghellini, C., Trigilio, C., \& Umana G. 1998, A\&A, 332, 251

Seward, F. D., Butt, Y. M., Karovska, M., Prestwich, A., Schlegel, E. M., \& Corcoran, M. 2001, ApJ, 553, 832

Townsley, L. K., et al. 2002a, Nucl. Instrum. Methods Phys. Res. A, 486, 716 Voges, W., et al. 1999, A\&A, 349, 389

Wegner, W. 1994, MNRAS, 270, 229

Wessolowski, U. 1996, in Röntgenstrahlung from the Universe, ed. H. U. Zimmerman et al. (MPE Rep. 263; Garching: MPE), 75

Yusef-Zadeh, F., \& Morris, M. 1987, ApJ, 320, 545

Zhao, J.-H., Desai, K., Goss, W. M., \& Yusef-Zadeh, F. 1993, ApJ, 418, 235

Zickgraf, F.-J., Wolf, B., Stahl, O., Leitherer, C., \& Appenzeller, I. 1986, A\&A, 163,119 